\title{
A Novel Current-Mode Low-Power Adjustable Wide Input Range Four- Quadrant Analog Multiplier
}

\author{
Siavash Mowlavia, $^{\mathrm{a},}$, Aram Baharmast ${ }^{\mathrm{b}}$, Jafar Sobhi ${ }^{\mathrm{a}}$, Ziadden Daei Koozehkanani ${ }^{\mathrm{a}}$
}

aDepartment of Electrical Engineering, University of Tabriz, Iran

${ }^{\mathrm{b}}$ ITEE Faculty, University of Oulu, Oulu, Finland

Abstract- In this paper, a novel current mode enhanced input range four-quadrant analog multiplier is presented. The proposed class $\mathrm{AB}$ based translinear current squarer allows the designer to control the input range via a digital code. Moreover, the proposed scheme improves the linearity of the circuit and has a high robustness against process variations. The Post-layout and Monte Carlo simulation of the multiplier in a $0.18 \mu \mathrm{m}$ standard CMOS technology shows an input range of $(1+\mathrm{m}) \times 10 \mu \mathrm{A}$ (where ' $\mathrm{m}$ ' is an adjustable parameter), a THD of 1\% (@1 MHz), a $3 \mathrm{~dB}$ bandwidth of $332.3 \mathrm{MHz}$ (for $\mathrm{m}=3$ ) and a power consumption of $186 \mu \mathrm{W}$.

13 Keywords- current mode, low-power, wide input range, analog multiplier, four-quadrant.

\section{Introduction}

Real-time analog multiplication of two signals is an important operation in the analog signal processing applications [1]. The multiplier is used in many analog signal processing systems such as modulators and mixers, variable gain amplifiers, adaptive filters, phase locked loops, artificial neural networks and fuzzy logic controllers $[1,2]$. A significant work for implementing analog multiplication has been conducted in CMOS and BJT mostly focusing on circuit level techniques. Analog voltage mode multipliers, which produce a voltage/current signal proportional to the multiplication of two input voltage signals have been categorized and analyzed in [1].

However, the voltage mode multipliers undergo various limitations regarding their direct dependence on the voltage supply and sensitivity to process variations. Furthermore, by scaling the technology and consequently scaling the supply voltage, the operating (input) range of this type of multipliers are being squeezed even more.

27 Current mode signal processing offers low voltage and high speed in comparison to voltage mode signal processing. However, the proposed current mode multipliers thus far, have a limited input range. Based on [3] analog current mode multiplication of two signals is generally realized either in weak inversion or strong inversion. Weak inversion multipliers are an interesting choice for extremely low voltage/power applications; however, they undergo limited input range and narrow

32 bandwidth [4-6].

\footnotetext{
${ }^{1}$ Corresponding author,

E-mail address: siavash.mowlavi@gmail.com
} 
Various implementations for CMOS current mode strong inversion multipliers have been proposed [7-15]. Fig.1 shows the block diagram of different realizations of the current mode multiplier in the strong inversion. One approach is to convert the input currents to voltage and then apply the voltage to two/three voltage mode squarers. This technique suffers from strong dependence of the output current to the device parameters and power supply variations [12-15]. Furthermore, the input ranges of these types of multipliers are limited by the maximum allowable voltage swing in the voltage squarer, which is generally limited to the voltage supply. In the second technique, different types of translinear loops (TL) (stacked or up-down configurations [16]) are used to realize the current mode squarer and/or geometric mean blocks as basic building blocks of the multiplier [7-11]. A TL is a special device arrangement that allows a useful large signal relationship among their currents [16-18]. The advantage of this technique over other abovementioned voltage or current mode techniques is in its capability to provide pure current mode output which is independent of device parameter or supply voltage. However, the input range of these types of multipliers is usually limited to the imposed limitation of the current squarer, which is the prerequisite of working in saturation region for all the transistors in the TLs. Moreover, the only way to enlarge input range in these types of multipliers is to increase the bias current, which in turn leads to increase in power.
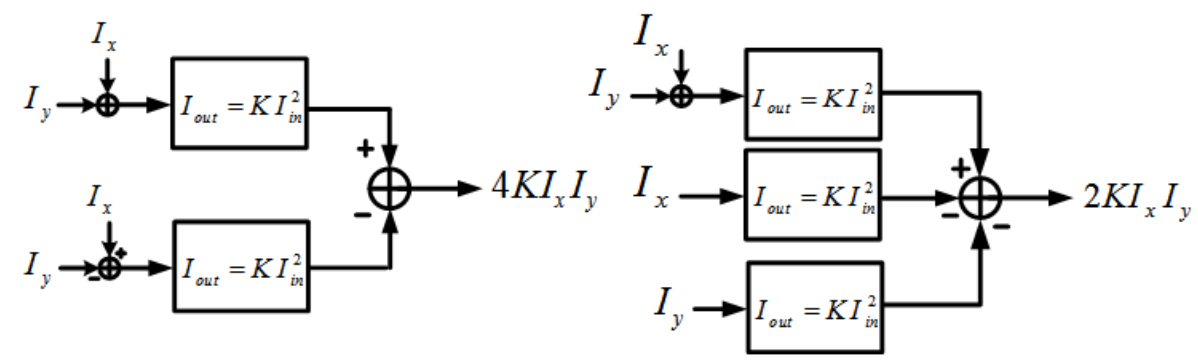

$$
K\left(I_{x}+I_{y}\right)^{2}-K\left(I_{x}-I_{y}\right)^{2}=4 K I_{x} I_{y}
$$

$$
K\left(I_{x}+I_{y}\right)^{2}-K I_{x}^{2}-K I_{y}^{2}=2 I_{x} I_{y}
$$

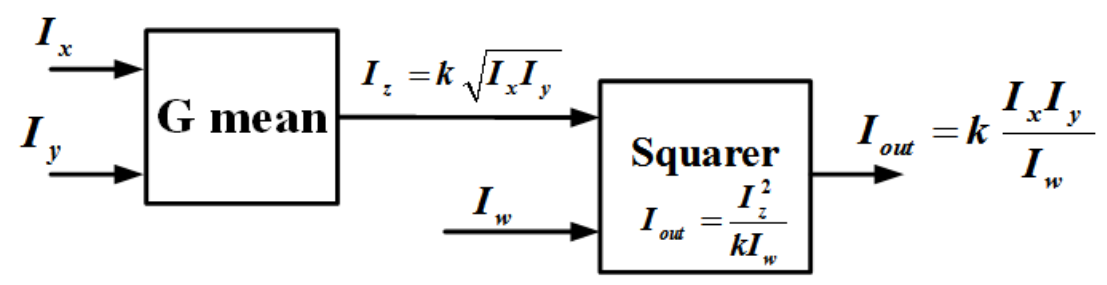

(b)

Fig. 1. Block diagram of different realizations of the current mode multiplier in the strong inversion

a) Using two/three squarers, b) Using geometric mean-squarer

The proposed multiplier offers a new solution to extend the input range without any increase in the power consumption. This solution combines modified low power class AB current buffer with translinear based current squarer to achieve a wide and adjustable input range multiplier that provides a pure current mode output. It is also shown that this technique improves the linearity of the multiplier. The paper is organized as follows. The proposed circuit is presented in Section 2, 
1 the proposed structure is analyzed in section 3, simulation results are shown in Section 4 and

2 conclusions are given in Section 5.

\section{Circuit Description}

4 The operation of the proposed multiplier is based on the implementation of the quadratic equation 5 as

$6 \quad I_{\text {out }}=k\left(I_{x}+I_{y}\right)^{2}-k\left(I_{x}-I_{y}\right)^{2}=4 k I_{x} I_{y}$

7 Where $I_{\text {out }}$ is the output current, $I_{x}$ and $I_{y}$ are input current signals and $k$ is the scaling factor. This 8 technique provides four quadrant operation in principle, while the geometric-mean based 9 technique is only capable of implementing two quadrant multiplication [7]. From (1) it is deduced 10 that two current mode squarers are needed to realize the current mode multiplication. These 11 squarers should provide two quadrant operation in order to achieve four quadrant multiplier. 12 Therefore, the characteristics of the current squarers (e.g. power consumption, input range and 13 linearity) directly determine that of the multiplier.

\subsection{Current-mode Squarer Circuit}

15 The current-mode squarer circuit is shown in Fig. 2. The circuit consists of two sub-blocks which 16 are highlighted in the figure: A modified class AB current buffer stage and a translinear loop 17 formed by $M_{1}-M_{4}$ which are biased in the saturation region [19]. The function of the class $\mathrm{AB}$ 18 buffer is to introduce the proper portion of the input current into the TL in such a way that the 19 whole circuit works as a two-quadrant current squaring circuit. The key point is that the input 20 current of the proposed circuit can be two quadrant without any need for extra bias currents thanks 21 to the intrinsic characteristic of the class AB stage. For the sourcing input currents, M11, M17, 22 M9, M7 conveys the current to the translinear loop and for the sinking input currents, M10, M16, 23 M8, M6 do the same. Moreover, it is shown in section 3.1 that the modified structure of the current 24 buffer helps to extend the input operating range of the squarer beyond the limitation dictated by 25 the current squarer. Furthermore, it is shown in the following sections that the proposed scheme 26 improves the linearity and robustness of the circuit against process variations. In the circuit the 27 aspect ratios of $M_{16}$ and $M_{17}$ (m-transistors) are ' $\mathrm{m}$ ' times larger than $M_{10}$ and $M_{11}$ respectively. 28 Applying KCL in the input node gives: 


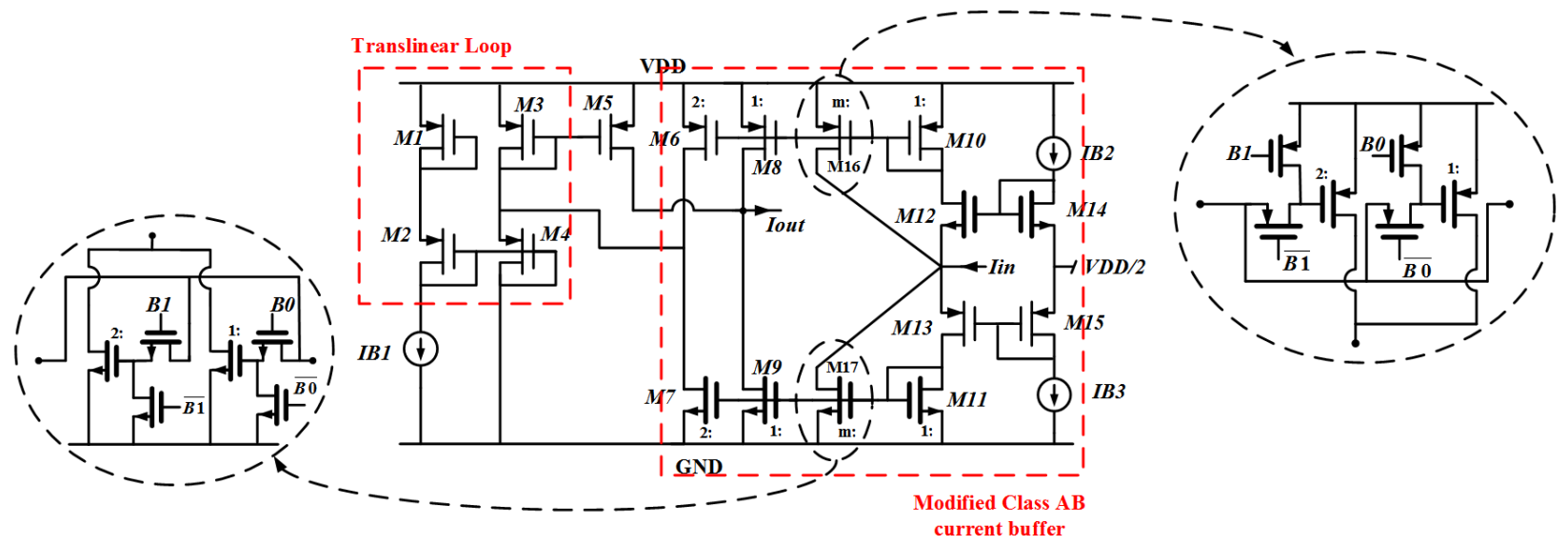

Fig. 2. The proposed Current-mode squarer

$3 \quad \mathrm{I}_{\text {in }}=\mathrm{I}_{M 16}+\mathrm{I}_{M 10}\left(\right.$ or: $\left.\mathrm{I}_{\text {in }}=\mathrm{I}_{M 17}+\mathrm{I}_{M 11}\right)$

4 The currents of transistors $M_{8}$ and $M_{10}$ are given as

$5 \quad \mathrm{I}_{M 8}=\mathrm{I}_{M 10}=\frac{I_{\text {in }}}{1+m} \quad\left(\right.$ or: $\left.: \mathrm{I}_{M 9}=\mathrm{I}_{M 11}=\frac{I_{\text {in }}}{1+m}\right)$

6 Based on the class $\mathrm{AB}$ operation, $M_{8}$ and $M_{9}$ provide two quadrant output current. This is also 7 valid for $M_{6}$ and $M_{7}$, which their aspect ratios are twice the diode connected transistors $M_{10}$ and $8 M_{11}$ respectively:

$9 \quad \mathrm{I}_{M 6}=\frac{2 I_{\text {in }}}{1+m}\left(\right.$ or: $\left.\mathrm{I}_{M 7}=\frac{2 I_{\text {in }}}{1+m}\right)$

10 These currents are injected into the TL in order to realize the two quadrant current squarer. Since 11 the aspect ratios of $M_{1}-M_{4}$ transistors are equal, based on the MOS translinearity (MTL) 12 principle in saturation region ([16])

$132 \sqrt{I_{B 1}}=\sqrt{I_{D S 3}}+\sqrt{I_{D S 4}}$

14 Where $I_{B 1}$ is the bias current of transistors $M_{1}$ and $M_{2} \cdot I_{D S 3}$ and $I_{D S 4}$ are the drain-source current 15 of transistors $M_{3}$ and $M_{4}$ respectively, which can be expressed as

$16 \quad I_{D S_{3}}=I_{D S_{5}}=I_{o u t}+\frac{I_{\text {in }}}{1+m}$

$17 \quad I_{D S_{4}}=I_{D S_{3}}-2 \frac{I_{\text {in }}}{1+m}=I_{\text {out }}-\frac{I_{\text {in }}}{1+m}$

18 Where $I_{D S 5}$ is the drain-source current of $M_{5}$ and $I_{\text {out }}$ is the output current of the squarer. 19 Substituting (6) and (7) into (5) yields 
$1 \quad 2 \sqrt{I_{B 1}}=\sqrt{I_{\text {out }}+\frac{I_{\text {in }}}{1+m}}+\sqrt{I_{\text {out }}-\frac{I_{\text {in }}}{1+m}}$

Squaring both sides gives the output current as

$3 \quad I_{\text {out }}=\frac{I_{\text {in }}{ }^{2}}{4 I_{B 1}(1+m)^{2}}+I_{B 1}$

4 Equation (9) describes the operation of the proposed squarer circuit. It is noteworthy that the output 5 current is insensitive to PVT variations thanks to the benefits of using TL based structure. Another 6 point is that ' $\mathrm{m}$ ' sets the amount of current injected into the TL. Therefore, with a larger $\mathrm{m}$, the 7 input range can be extended significantly. The implementation of ' $\mathrm{m}$ ' is realized through a set of 8 switches that can be controlled by a digital code, as it is shown in Fig.2 and is discussed in more 9 details in sections 2.2 and 3.1 .

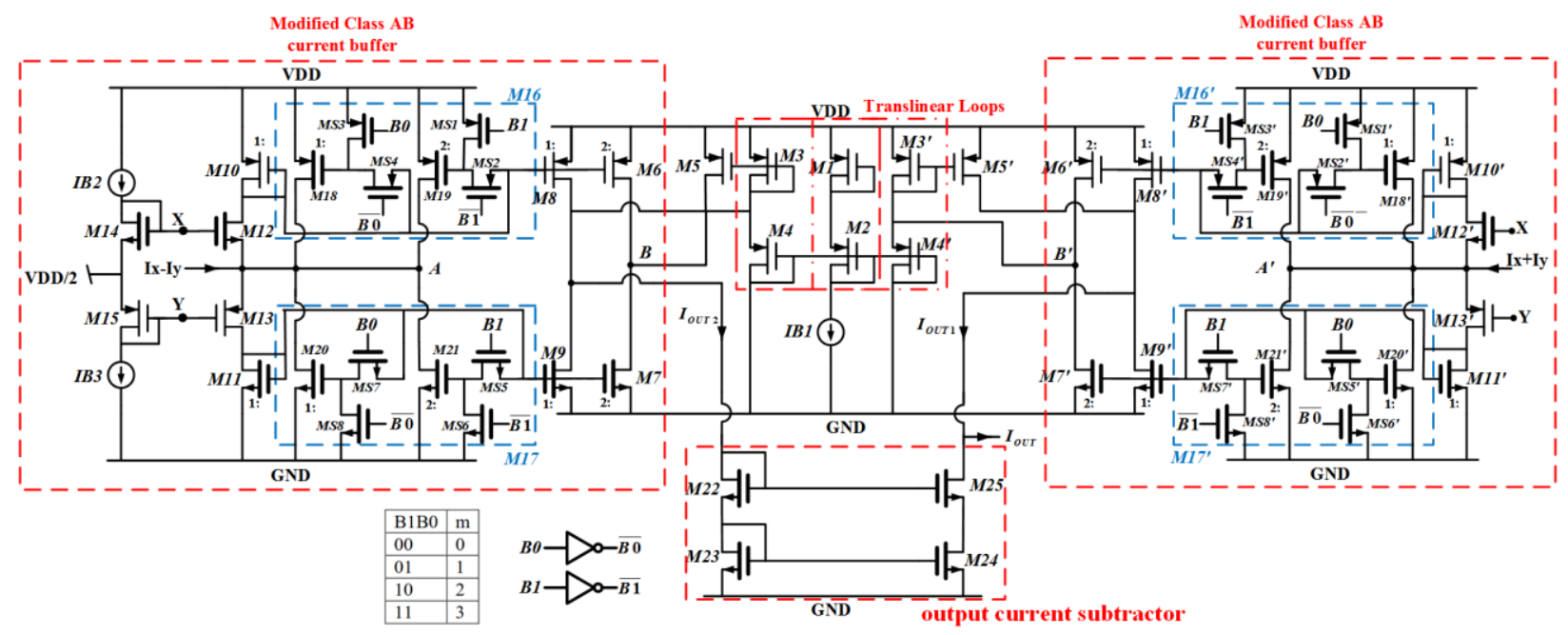

Fig. 3. The proposed four-quadrant multiplier

\subsection{Current-mode Multiplier Circuit}

13 The proposed current mode multiplier is shown in Fig. 3. It consists of two current mode squarers as discussed in section 2.1 and a current subtractor. Each current squarer comprises a class $\mathrm{AB}$ current buffer and a TL. In order to implement m-transistors $\left(M_{17}, M_{17}{ }^{\prime}, M_{16}\right.$ and $\left.M_{16}{ }^{\prime}\right)$ two binary weighted transistors and a set of MOS switches are used. For instance, the $M_{17}$ is implemented by transistors $M_{20}(: 1)$ and $M_{21}(: 2)$ and MOS switches of $M_{s 5}, M_{s 6}, M_{s 7}$ and $M_{s 8}$. In a similar manner, $M_{16}$ is implemented by transistors $M_{18}(: 1)$ and $M_{19}(: 2)$ and MOS switches of $M_{s 1}, M_{s 2}$, $M_{s 3}$ and $M_{s 4}$. A 2-bit binary code $\left(B_{1} B_{0}\right)$ controls these switches to provide four values of ' $\mathrm{m}$ ' $(0$, 1,2 or 3). It is shown in the following sections that selecting higher amounts of ' $\mathrm{m}$ ' leads to improve the input range and linearity of the multiplier. The two TLs $\left(M_{1}, M_{2}, M_{3}, M_{4}\right.$ and $\left.M_{1}, M_{2}, M_{3}{ }^{\prime}, M_{4}{ }^{\prime}\right)$ share $M_{1}$ and $M_{2}$, which are biased by a constant current and provide a 
1 constant bias point for the TLs. Using this technique, the power consumption of the TLs is reduced

2 and the device mismatches between TL transistors and the biased currents are alleviated. $M_{14}$ and

$3 \quad M_{15}$ are also shared between two class AB current mirrors in the same manner. The input signal

4 of the right squarer is $I_{X}+I_{Y}$ while $I_{X}-I_{Y}$ is applied to the left one. In current-mode, the

5 summation and subtraction of two signals can be simply implemented by a combination of simple

6 or class $\mathrm{AB}$ current mirrors as in [11]. According to (9) the output current of each squarer is

$$
\begin{aligned}
& I_{\text {out } 1}=\frac{\left(I_{X}+I_{Y}\right)^{2}}{4 I_{B 1}(1+m)^{2}}+I_{B 1} \\
& I_{\text {out } 2}=\frac{\left(I_{X}-I_{Y}\right)^{2}}{4 I_{B 1}(1+m)^{2}}+I_{B 1}
\end{aligned}
$$

7 The current subtractor which is a cascade current mirror $\left(M_{22}-M_{25}\right)$ removes the DC and other

8 squared terms. Therefore, the output current of the circuit is derived as

$$
I_{\text {out }}=I_{\text {out } 2}-I_{\text {out } 1}=\frac{I_{X} I_{Y}}{I_{B 1}(1+m)^{2}}
$$

9 Equation (12) indicates the function of the proposed current-mode multiplier and demonstrates

10 that the output current is independent of process parameters. Parameter ' $\mathrm{m}$ ' which is controllable

11 via a 2-bit binary code $\left(B_{1} B_{0}\right)$, determines the size of $M_{16}$ and $M_{17}$ and plays a key role in

12 controlling the input range of the multiplier without an increase in power consumption. Therefore

13 this structure allows obtaining wider input range by selecting larger ' $\mathrm{m}$ '. Moreover, it is shown in

14 sections 3.2, 3.3 and 3.4 that increasing ' $\mathrm{m}$ ' leads to alleviate the absolute error causing by the

15 different types of mismatches ( $V_{T H}$ and transconductance parameter mismatches) and improves

16 the linearity of the circuit.

\section{3. Performance Analysis}

\subsection{Input Range}

19 The input range of the multiplier is basically restricted by the prerequisite of working in saturation

20 for transistors of translinear loops. Based on this, the input range of the proposed multiplier can be

21 derived as

$22 \quad \frac{\left|I_{X}+I_{Y}\right|}{1+m} \leq 2 I_{B} \quad, \frac{\left|I_{X}-I_{Y}\right|}{1+m} \leq 2 I_{B}$

23 Equation 13 yields in

$24 \quad\left|I_{X}\right|=\left|I_{Y}\right| \leq(1+m) I_{B}$ 
1 In the conventional current mode multipliers such as [3], [8,9], [11], [15], the input range of the 2 circuit is extended by increasing the bias current which leads to a higher power consumption. 3 However, equation (14) indicates that the maximum allowable input current of the proposed 4 multiplier can be adjusted as a function of $\mathrm{m}$, without the need for extra power consumption. The 5 implementation of this parameter can be done by controlling the sizes of $M_{16}$ and $M_{17}$ in each 6 squarer. It is shown in Fig. 3 for four different amounts of ' $\mathrm{m}$ ' (0-3) using NMOS and PMOS 7 switches and two binary bits, as it is discussed in section 2.2.

\section{2. $\quad \mathrm{V}_{\mathrm{TH}}$ Mismatch}

9 The body-source voltage of a MOS transistor affects the threshold voltage by [20]

$V_{T H}=V_{T H 0}+\gamma\left(\sqrt{\left|2 \Phi_{F}+V_{S B}\right|}-\sqrt{\left|2 \Phi_{F}\right|}\right)$

11 Where, $V_{T H 0}$ is the threshold voltage with $V_{S B}=0, \gamma$ is the body effect coefficient and $2 \Phi_{F}$ is the 12 substrate bias. This affect can be cancelled out if the bulk terminal is tied to the source. In the 13 proposed circuit n-well CMOS technology is used therefore all PMOS bodies are tied to their 14 source while all NMOS bodies are tied to the ground. Since all transistors in translinear loops are 15 PMOS, the body effects of these transistors are completely eliminated. However, the fabrication 16 process may cause a $V_{T H}$ mismatch between these transistors. The $V_{T H}$ mismatches of the $M_{1}-M_{4}$ 17 are modeled as follows

$V_{T H, i}=\overline{V_{T H}}\left(1+\delta_{i}\right) \quad \mathrm{i}=1-4$

19 Where $V_{T H, i}$ is the threshold voltage of the $\mathrm{i}^{\text {th }}$ transistor and $\overline{V_{T H}}$ is the average threshold voltage.

20 The MTL principle in the presence of $V_{T H}$ mismatches can be written as

$21 \sqrt{\frac{I_{B}}{K}}+\overline{V_{T H}}\left(1+\delta_{1}\right)+\sqrt{\frac{I_{B}}{K}}+\overline{V_{T H}}\left(1+\delta_{2}\right)=\sqrt{\frac{I_{\text {OUT }}+\frac{I_{\text {in }}}{1+m}}{K}}+\overline{V_{T H}}\left(1+\delta_{3}\right)+$

$22 \sqrt{\frac{I_{\text {OUT }}-\frac{I_{\text {in }}}{1+m}}{K}}+\overline{V_{T H}}\left(1+\delta_{4}\right)$

23 Where $K$ is the transconductance parameters of the TL transistors. Squaring both sides and 24 neglecting $\delta^{2}$ terms, the output current of the squaring circuit is

$25 I_{O U T}=\frac{I_{i n}{ }^{2}}{4 I_{B 1}(1+m)^{2}}\left(1-\sqrt{\frac{K}{I_{B 1}}} \overline{V_{T H}}\left[\left(\delta_{1}+\delta_{2}\right)-\left(\delta_{3}+\delta_{4}\right)\right]\right)+I_{B 1}+\sqrt{K I_{B 1}} \overline{V_{T H}}\left[\left(\delta_{1}+\delta_{2}\right)-\left(\delta_{3}+\delta_{4}\right)\right]$

26 Equation (18) is obtained using the following approximation

$27 \frac{1}{1+x} \approx 1-x$ for $|x| \ll 1$ 
1 Hence the output current of the multiplier can be derived as follows

$2 \quad I_{O U T}=\frac{I_{X} I_{Y}}{I_{B 1}(1+m)^{2}}\left(1-\sqrt{\frac{K}{I_{B 1}}} \overline{V_{T H}}\left[\left(\delta_{1}+\delta_{2}\right)-\left(\delta_{3}+\delta_{4}\right)\right]\right)$

3 Therefore the absolute output error of the multiplier resulting from threshold voltage mismatch is

$4 \quad \mid$ Error $\mid=\frac{I_{X} I_{Y}}{I_{B 1}(1+m)^{2}}\left(\sqrt{\frac{K}{I_{B 1}}} \overline{V_{T H}}\left[\left(\delta_{1}+\delta_{2}\right)-\left(\delta_{3}+\delta_{4}\right)\right]\right)$

5 Equation (21) indicates that introducing ' $\mathrm{m}$ ' to the circuit reduces the absolute error due to $V_{T H}$

6 mismatches.

$7 \quad$ 3.3. Transconductance Parameter Mismatch

8 The effect of transconductance parameter mismatches is analyzed in two parts: The mismatch

9 effect of the transistors in the TL and the mismatch effect of the class AB current buffers.

\subsubsection{Transconductance parameters mismatch in TLs}

11 The mismatch of the transconductance parameters of the TLs are modeled as follows:

$12 \quad K_{i}=\bar{K}\left(1+\sigma_{i}\right) \quad \mathrm{i}=1-4$

13 Where $K_{i}$ is the transconductance of the $\mathrm{i}^{\text {th }}$ transistor and $\bar{K}$ is the nominal value of the

14 transconductance. In this case, the MTL principle can be written as

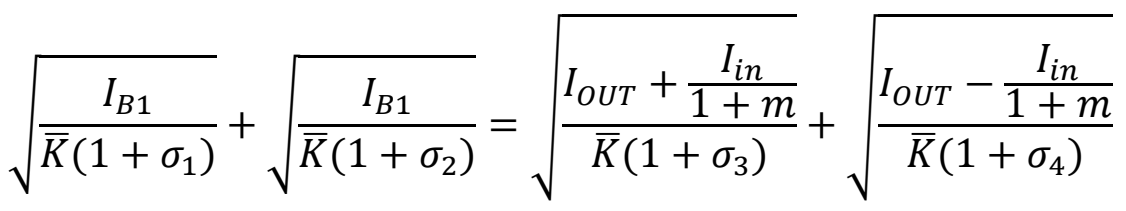

16 Squaring both sides, neglecting $\sigma^{2}$ terms and using the approximation $\sqrt{1+x}=1+\frac{1}{2} x,|x| \ll 1$,

17 the output current of the squaring circuit is given by

$18 I_{\text {OUT }}=\frac{I_{\text {in }}{ }^{2}}{4 I_{B 1}(1+m)^{2}}\left(1+\frac{\sigma_{1}+\sigma_{2}-\sigma_{3}-\sigma_{4}}{2}\right)+I_{B 1}\left(1+\frac{\sigma_{3}+\sigma_{4}-\sigma_{1}-\sigma_{2}}{2}\right)+$

$19 \frac{I_{\text {in }}}{4(1+m)}\left(\sigma_{3}-3 \sigma_{4}\right)-\frac{I_{\text {in }}{ }^{3}}{16 I_{B 1}{ }^{2}(1+m)^{3}}\left(\sigma_{3}+\sigma_{4}\right)$

20 Thus the output current of the multiplier in presence of the transconductance mismatches and the

21 resulted absolute error are as follows 
$1 \quad I_{\text {OUT }}=\frac{I_{X} I_{Y}}{I_{B 1}(1+m)^{2}}\left(1+\frac{\sigma_{1}+\sigma_{2}-\sigma_{3}-\sigma_{4}}{2}\right)+\frac{I_{Y}}{2(1+m)}\left(\sigma_{3}-3 \sigma_{4}\right)-\frac{I_{Y}{ }^{3}+3 I_{X}{ }^{2} I_{Y}}{8 I_{B 1}{ }^{2}(1+m)^{3}}\left(\sigma_{3}+\sigma_{4}\right)$

$2 \quad \mid$ Error $\mid=\frac{I_{X} I_{Y}}{I_{B 1}(1+m)^{2}}\left(\frac{\sigma_{3}+\sigma_{4}-\sigma_{1}-\sigma_{2}}{2}\right)+\frac{I_{Y}}{2(1+m)}\left(3 \sigma_{4}-\sigma_{3}\right)+\frac{I_{Y}{ }^{3}+3 I_{X}{ }^{2} I_{Y}}{8 I_{B 1}{ }^{2}(1+m)^{3}}\left(\sigma_{3}+\sigma_{4}\right)$

3 From (26), the absolute error due to the transconductance parameters mismatches in TLs is 4 diminished with the increase of ' $m$ '.

$5 \quad$ 3.3.2 Transconductance parameters mismatch in class $A B$ current buffers

6 Assume there are mismatches between transconductance parameters of PMOS (or NMOS) 7 transistors in the class $\mathrm{AB}$ current buffers in Fig.1. In this case

$8 \quad K_{i}=\bar{K}_{i}\left(1+\varepsilon_{i}\right) \quad \mathrm{i}=6,9,10,16$

9 Where $\bar{K}_{i}$ is a nominal value for $\mathrm{i}^{\text {th }}$ transistor. Therefore, the drain-source current of $\mathrm{M}_{16}$ is

$10 \quad I_{D S_{16}}=\frac{K_{16}}{K_{10}} I_{D S_{11}}=\frac{\bar{K}\left(1+\varepsilon_{16}\right)}{\bar{K}\left(1+\varepsilon_{10}\right)} I_{D S_{10}}$

11 Assuming $\varepsilon_{i} \ll 1$, using (18) and neglecting second order terms yields in

$12 I_{D S_{16}}=m\left(1+\varepsilon_{16}-\varepsilon_{10}\right) I_{D S_{10}}$

13 Substituting (29) into (2), the drain-source current of $M_{10}$ is given by

$14 \quad I_{D S_{10}}=\frac{I_{\text {in }}}{1+m}\left(1-\frac{m}{1+m}\left(\varepsilon_{16}-\varepsilon_{10}\right)\right)$

15 From (27) and (30), the drain-source currents of $M_{8}$ and $M_{6}$ are given by

$16 I_{D S_{8}}=\frac{I_{\text {in }}}{1+m}\left(1-\frac{m}{1+m} \varepsilon_{16}-\frac{1}{1+m} \varepsilon_{10}+\varepsilon_{8}\right)$

$17 \quad I_{D S_{6}}=\frac{2 I_{\text {in }}}{1+m}\left(1-\frac{m}{1+m} \varepsilon_{16}-\frac{1}{1+m} \varepsilon_{10}+\varepsilon_{6}\right)$

18 Injecting these currents into the TL transistors $\left(M_{1}-M_{4}\right)$ and calculating the output current of

19 squaring circuit yields

$20 \quad I_{\text {out }}=\frac{I_{\text {in }}{ }^{2}}{4 I_{B 1}(1+m)^{2}}+I_{B 1}-\frac{I_{\text {in }}}{1+m}\left(\varepsilon_{8}-\varepsilon_{6}\right)$

21 Therefore the output current of the multiplier and the generated error can be expressed as follows 


$$
\begin{aligned}
& 1 \quad I_{\text {out }}=\frac{I_{X} I_{Y}}{I_{B 1}(1+m)^{2}}-\frac{2 I_{Y}}{1+m}\left(\varepsilon_{8}-\varepsilon_{6}\right) \\
& 2 \quad \mid \text { Error } \mid=\frac{2 I_{Y}}{1+m}\left(\varepsilon_{8}-\varepsilon_{6}\right)
\end{aligned}
$$

3 From (26) and (35) it can be seen that the absolute error introduced due to transconductance

4 parameter mismatches is reduced by increasing ' $m$ '. Furthermore (35) indicates that $M_{10}$ and $M_{16}$

5 mismatches have no effects on the output current and the mismatch error is only proportional to

6 the amplitude of the $I_{Y}$.

\section{3.4. Total Harmonic Distortion (THD)}

8 The two main sources of non-ideality in the proposed multiplier are the mismatches of the

9 transistors and the channel-length modulation error of mirror transistors which affect the linearity 10 of the circuit [21] (due to the fact that the TL transistors are diode connected, the channel length 11 modulation effect would be waived for these transistors). Since the effect of $V_{T H}$ and 12 transconductance parameter mismatches is discussed in section 3.2 and 3.3, in the following 13 expressions, it is supposed that there is a complete matching between the current mirror transistors 14 (M9 \& M11) and (M8 \& M10) in the modified class AB current buffer (Fig.2). Taking into account 15 the channel modulation effect for the NMOS side

$$
16 \frac{i_{11}}{1+\lambda V_{D S 11}}=\frac{i_{9}}{1+\lambda V_{D S 9}}
$$

17 Since $V_{D S 11}=V_{G S 11}(36)$ can be rewritten as

$$
18 \frac{i_{11}}{1+\lambda\left(V_{T H^{+}} \sqrt{\frac{i_{11}}{K_{N}}}\right)}=\frac{i_{9}}{1+\lambda V_{D D} / 2}
$$

$19 \frac{i_{9}}{i_{11}}=\frac{1+\lambda_{N} V_{D D} / 2}{1+\lambda_{N}\left(V_{T H^{+}}+\sqrt{\frac{i_{11}}{K_{N}}}\right)}$

20 Where $V_{D D} / 2$ is the dc voltage at the drain of $M_{9}$. Taking the derivative of $i_{9}$ with respect to $i_{11}$ 21 gives

$22 \frac{\partial i_{9}}{\partial i_{11}}=\left(1+\frac{\lambda_{N} V_{D D}}{2}\right) \frac{1+\lambda_{N}\left(V_{T H}+\frac{1}{2} \sqrt{\frac{i_{11}}{K_{N}}}\right)}{\left(1+\lambda_{N}\left(V_{T H^{+}}+\sqrt{\left.\left.\frac{i_{11}}{K_{N}}\right)\right)^{2}}\right.\right.} \cong 1-\lambda_{N}\left(V_{T H}-\frac{V_{D D}}{2}+\frac{3}{2} \sqrt{\frac{i_{11}}{K_{N}}}\right)$

23 Where the terms including $\lambda_{N}^{2}$ are neglected and it is assumed $2 \lambda_{N}\left(V_{T H}+\sqrt{i_{11} / K_{N}}\right) \ll 1$. 24 Following the same steps for the P-type current mirror (M8-M10) gives 
$1 \quad \frac{\partial i_{8}}{\partial i_{10}}=1-\lambda_{P}\left(V_{T H}-\frac{V_{D D}}{2}+\frac{3}{2} \sqrt{\frac{i_{8}}{K_{P}}}\right)$

2 Assuming the transconductance gain to be equal for both current mirrors $\left(K_{N}=K_{P}=K\right)$, the

3 second and third harmonic distortion are calculated as ([21]):

$4 \quad H D 2=\frac{1}{8}\left(\frac{\partial i_{9}}{\partial i_{11}}-\frac{\partial i_{8}}{\partial i_{10}}\right)_{i_{i n}=I_{M}}=\frac{\left(\lambda_{N}-\lambda_{P}\right)}{8}\left(\frac{V_{D D}}{2}-V_{T H}-\frac{3}{2} \sqrt{\frac{I_{M}}{K(m+1)}}\right)$

$5 \quad H D 3=\frac{1}{24}\left[\left(\frac{\partial i_{9}}{\partial i_{11}}+\frac{\partial i_{8}}{\partial i_{10}}\right)_{i_{1}=I_{M}}-\left(\frac{\partial i_{9}}{\partial i_{11}}+\frac{\partial i_{8}}{\partial i_{10}}\right)_{i_{1}=I_{Q}}\right] \cong \frac{\left(\lambda_{N}+\lambda_{P}\right)}{16} \sqrt{\frac{I_{Q}}{K}}\left(1-\sqrt{\frac{I_{M}}{(m+1) I_{Q}}}\right)$

6 Where $I_{M}$ is the maximum amplitude of the input current and $I_{Q}$ is the quiescent current of the 7 class AB buffer. (41) and (42) indicate that both HD2 and HD3 are decreased by increasing ' $\mathrm{m}$ '.

\section{Simulation Results}

9 The performance of the circuit is simulated using Cadence by $0.18 \mu \mathrm{m}$ standard CMOS technology.

10 A supply voltage of $1.8 \mathrm{~V}$ is used and $I_{B 1}$ is set to $10 \mu \mathrm{A}$ while $I_{B 2}$ and $I_{B 3}$ are set to $1.5 \mu \mathrm{A}$. All

11 current sources have been implemented using simple current mirrors. The device dimensions of 12 the proposed multiplier are shown in Table I. The power consumption of the proposed circuit is

$13186 \mu \mathrm{W}$. The layout of the proposed multiplier is shown in Fig. 4 in which the occupied area is $141590 \mu \mathrm{m}^{2}$.

Table 1. Device dimensions of the proposed multiplier

\begin{tabular}{|c|c|}
\hline Transistors & Aspect ratios $(\mu \mathrm{m} / \mu \mathrm{m})$ \\
\hline$M_{1}-M_{5}$ & $2 / 0.5$ \\
\hline$M_{6}$ & $6 / 1$ \\
\hline$M_{7}$ & $8 / 1$ \\
\hline$M_{8}, M_{10}$ & $3 / 1$ \\
\hline$M_{9}, M_{11}$ & $4 / 1$ \\
\hline$M_{12}, M_{14}$ & $2 / 1$ \\
\hline$M_{13}, M_{15}$ & $5 / 0.5$ \\
\hline$M_{18}$ & $3 / 1$ \\
\hline$M_{19}$ & $6 / 1$ \\
\hline$M_{20}$ & $4 / 1$ \\
\hline$M_{21}$ & $8 / 1$ \\
\hline$M_{22}-M_{25}$ & $5 / 0.18$ \\
\hline$M_{s 1}-M_{s 8}$ & $0.5 / 0.18$ \\
\hline
\end{tabular}




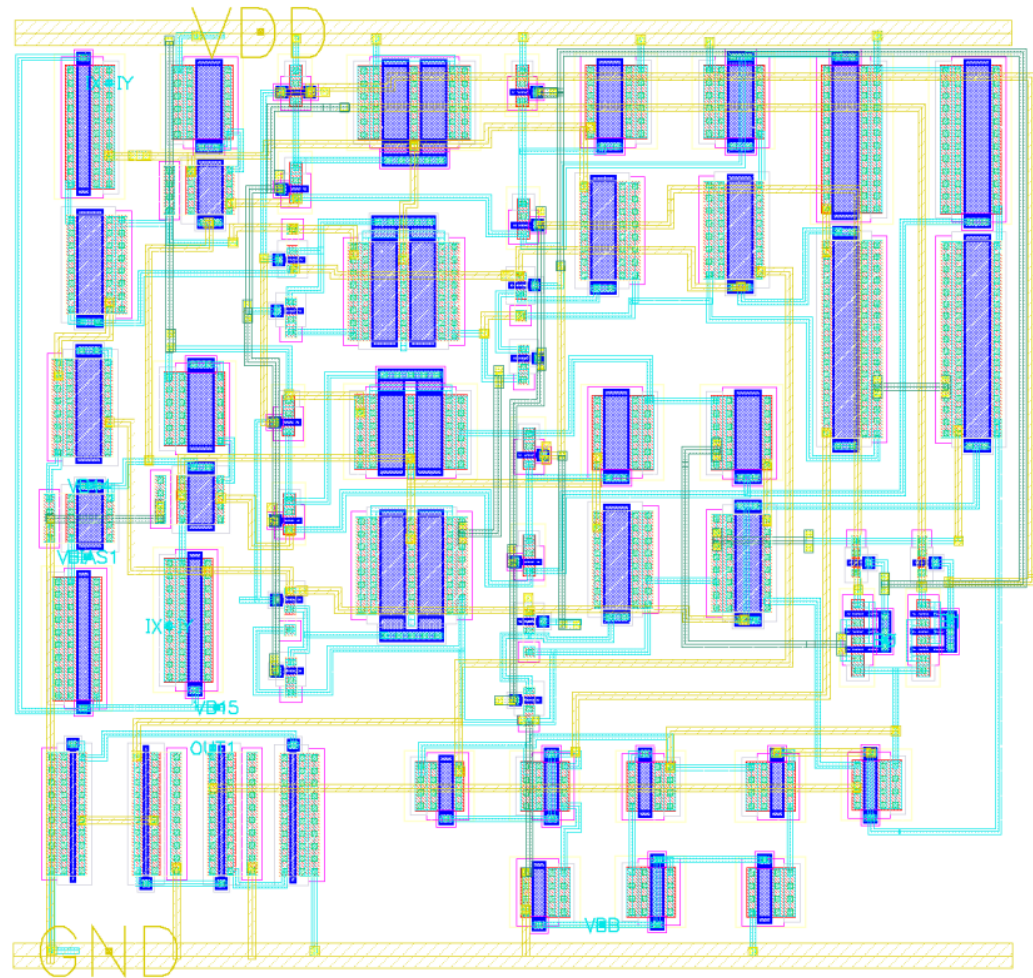

Fig. 4. Layout of the proposed current-mode multiplier

3 Fig. 5 illustrates the transient response of the multiplier for various amounts of ' $\mathrm{m}$ ' and 4 demonstrates the functionality of the circuit as an amplitude modulator for different input 5 amplitudes. For each value of ' $\mathrm{m}$ ' the input currents $\left(I_{X}\right.$ and $\left.I_{Y}\right)$ are set to the maximum allowable 6 amplitude at $1 \mathrm{MHz}$ and $100 \mathrm{KHz}$ frequencies respectively. For $\mathrm{m}=0,1,2$ and 3, the input currents 7 are set to $10 \mu A, 20 \mu A, 30 \mu A$ and $40 \mu A$ respectively. Therefore, the maximum amplitude of the 8 output current for all values of ' $\mathrm{m}$ ' would be equal to $10 \mu \mathrm{A}$ based on equation (12). Thus, the 9 output current of the circuit for different amounts of ' $\mathrm{m}$ ' should be similar as it is shown in Fig. 5. 10 It is noteworthy that the only difference between $m=0,1,2$ and 3 is the difference between their 11 input ranges. For instance, the input range for $\mathrm{m}=1$ is $20 \mu \mathrm{A}$ which is two times bigger than the 12 input range of $\mathrm{m}=0(10 \mu A)$ while the output currents are the same for the two cases. Similarly, the 13 input ranges for $\mathrm{m}=2$ and $\mathrm{m}=3$ are equal to $30 \mu \mathrm{A}$ and $40 \mu \mathrm{A}$ respectively while there is no 14 difference between their transient outputs.

15 Fig. 6 shows the DC transfer characteristic of the output current versus the input currents. For each 16 amount of ' $\mathrm{m}$ ', the first input is swept between $\pm(m+1) \times 10 \mu A$ while a maximum allowable 17 DC current is fed to the second input. For instance, for $\mathrm{m}=0$ the input current is swept between $1810 \mu A$ to $+10 \mu A$ while for $\mathrm{m}=3$ it is swept between $-40 \mu A$ to $+40 \mu A$. It can be seen that the circuit 19 works perfectly in the whole operating range for various amounts of ' $\mathrm{m}$ '. 

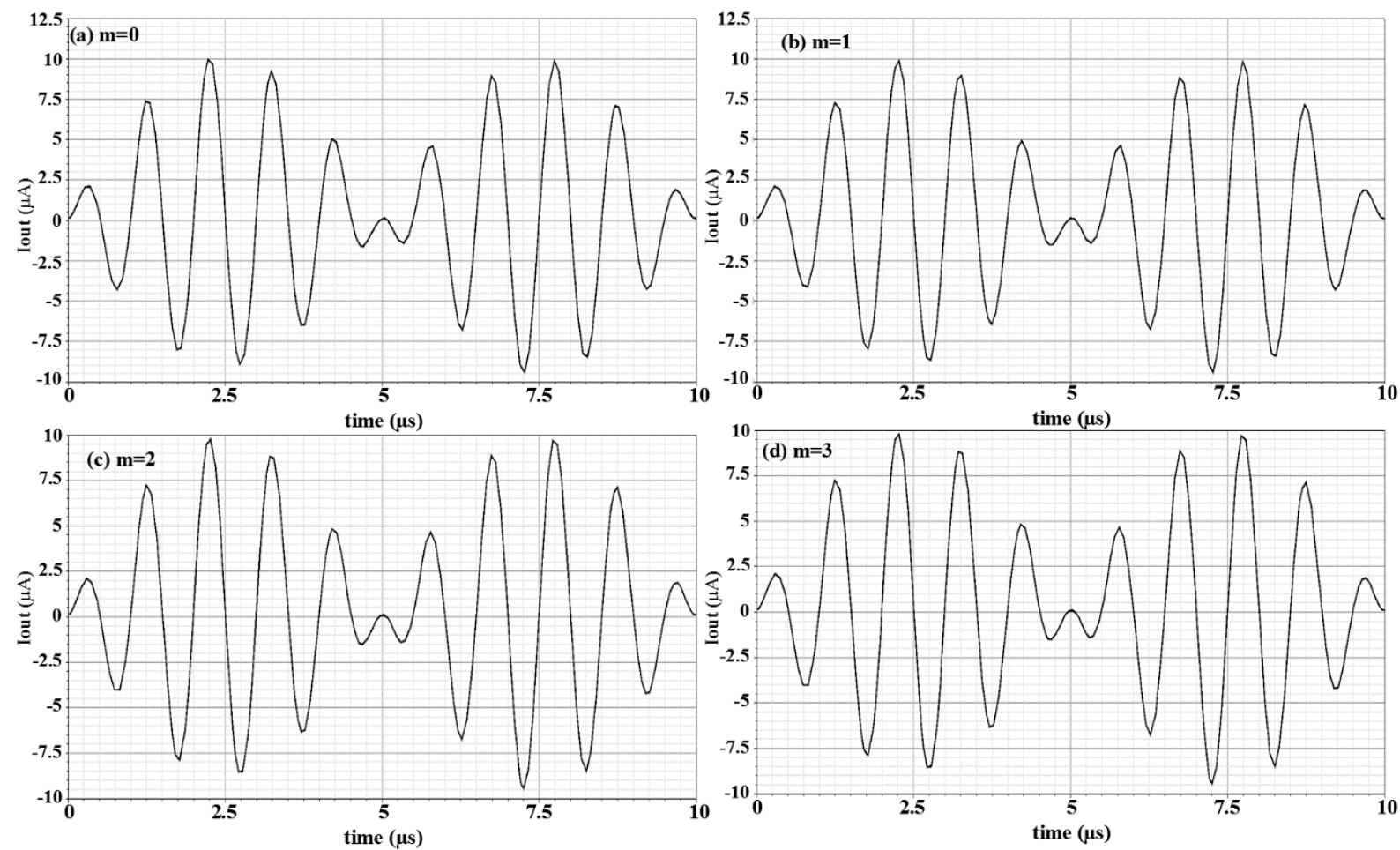

Fig. 5. Post-layout simulated Time response of the proposed multiplier for four different amounts of $m$, $I_{x}=(m+1) \times 10 \sin \left(2 \pi \times 10^{6}\right) \mu A, I_{y}=(m+1) \times 10 \sin \left(2 \pi \times 10^{5}\right) \mu A$ a) $\mathrm{m}=0 \mathrm{~b}) \mathrm{m}=1 \mathrm{c}) \mathrm{m}=2 \mathrm{~d}) \mathrm{m}=3$
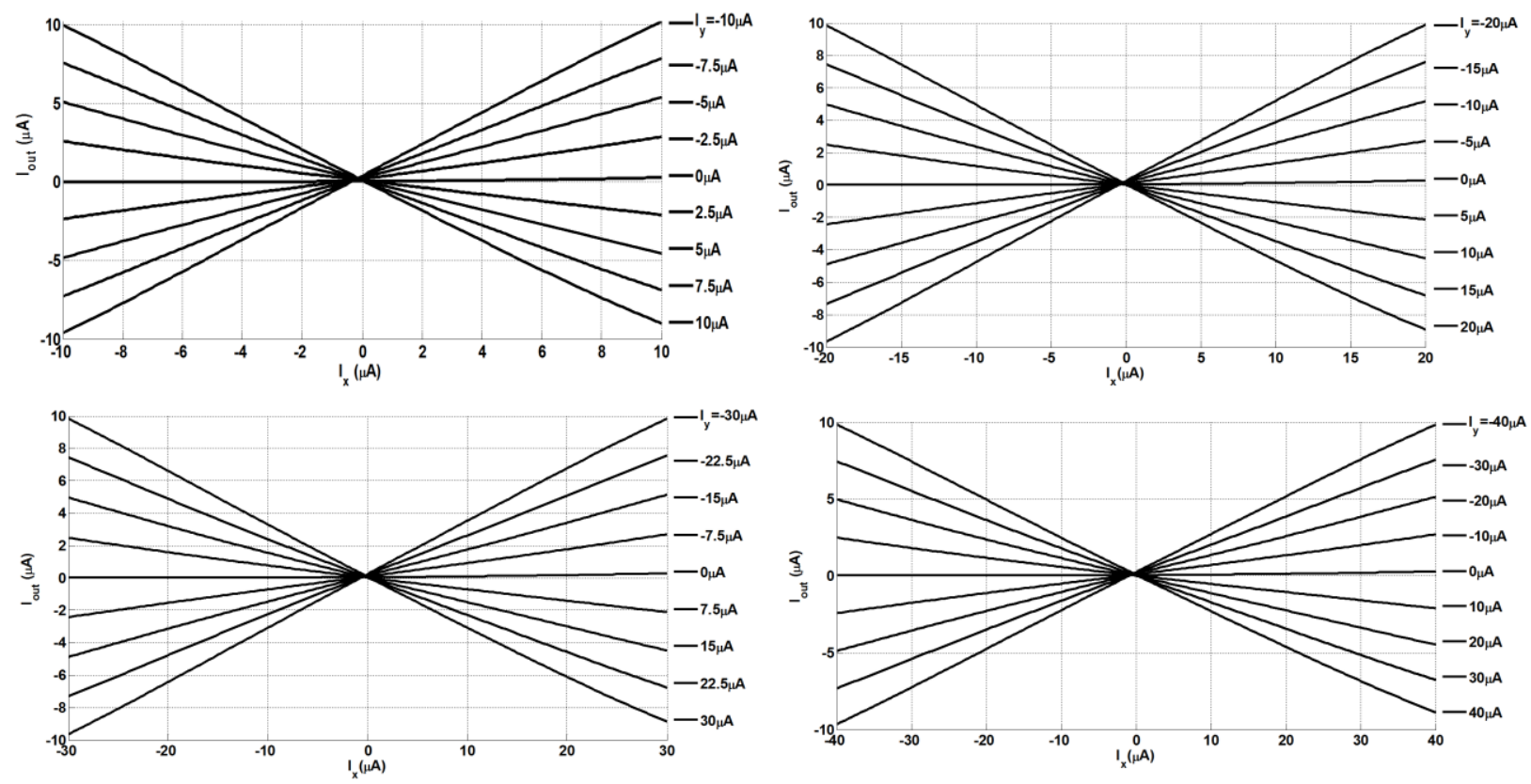

Fig. 6. Post-layout simulated DC transfer function of proposed multiplier a) $m=0$ b) $m=1$ c) $m=2 d) m=3$

6 Fig. 7 shows total harmonic distortion (THD) in three input frequencies of 1, 20 and $50 \mathrm{MHz}$ 
versus the amplitude of the first input signal $\left(I_{X}\right)$. Here $I_{X}$ is a sinusoidal signal with an amplitude of $(m+1) \times 10 \mu A$ while a same amount of DC current is fed to the $I_{Y}$. The THD of the circuit in $1 \mathrm{MHz}$ frequency is $\sim 1 \%$ for all amounts of ' $\mathrm{m}$ ' and it lowers down from $\sim 8 \%$ (for $\mathrm{m}=1, I_{X}=$ $10 \mu \mathrm{A}$ ) to less than $1.2 \%$ (for $\mathrm{m}=3, I_{X}=10 \mu \mathrm{A}$ ) at a high frequency of $50 \mathrm{MHz}$. Fig. 7 verifies that increasing ' $\mathrm{m}$ ' improves the THD of the circuit as it is predicted in section 3.4 .
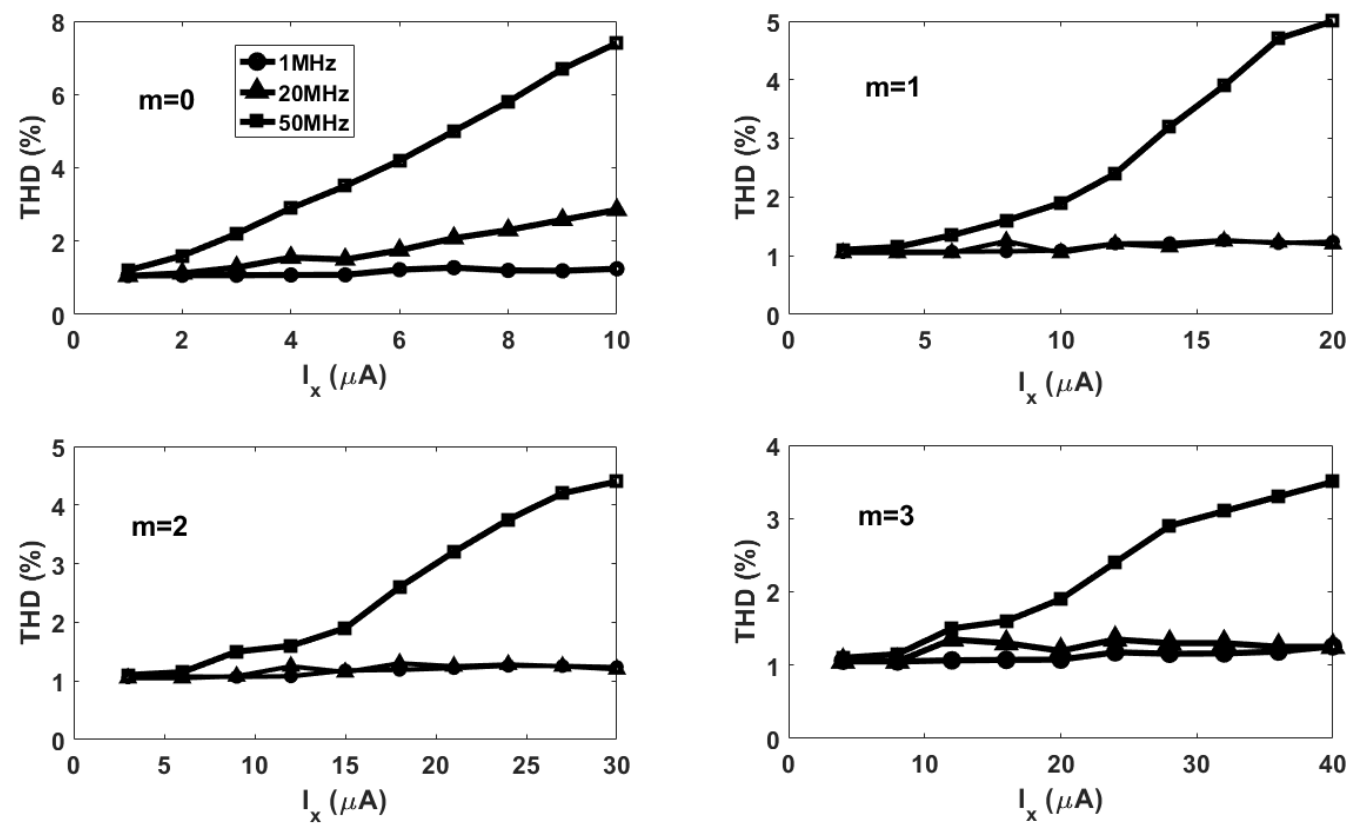

Fig. 7. Post-layout simulated Total harmonic distortion versus input current $\left(I_{Y_{D C}}=(m+1) \times 10 \mu A\right)$

Fig. 8 shows the frequency response of the proposed circuit. In this figure, $I_{X}$ is small signal and $I_{Y}$ is set to $10 \mu \mathrm{A}, 20 \mu \mathrm{A}, 30 \mu \mathrm{A}$ and $40 \mu \mathrm{A}$ for $\mathrm{m}=0,1,2$ and 3 respectively. The $-3 \mathrm{~dB}$ bandwidth of proposed multiplier varies from $253.4 \mathrm{MHz}$ for $\mathrm{m}=0$ to $332.3 \mathrm{MHz}$ for $\mathrm{m}=3$.

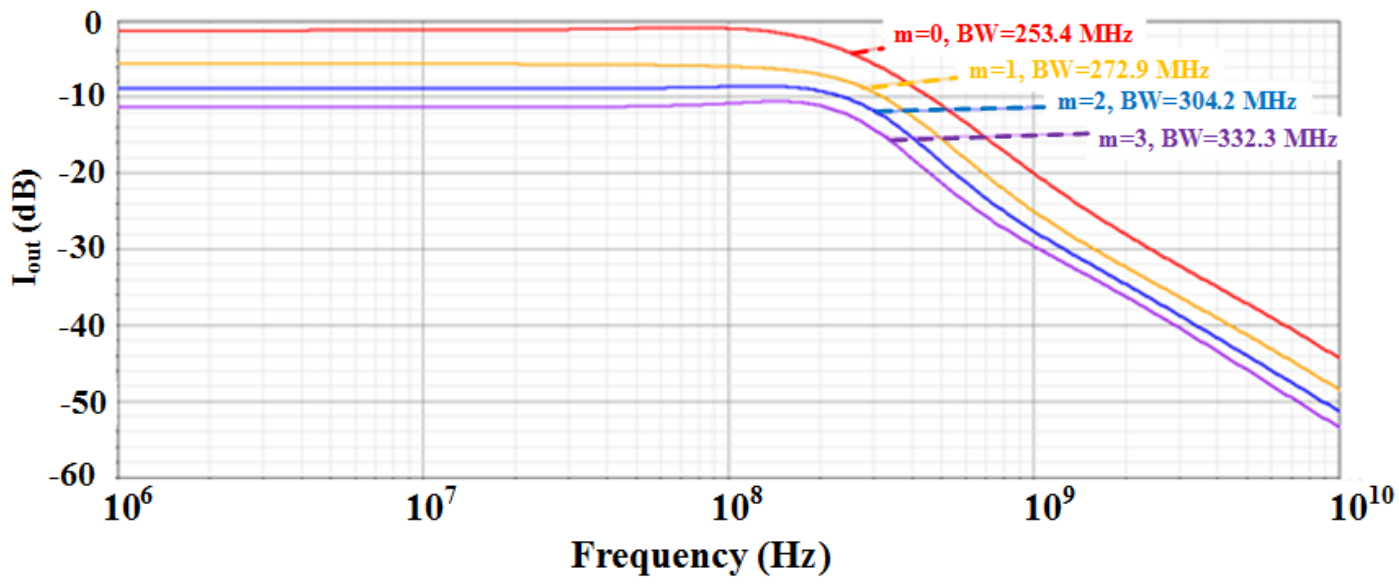

Fig. 8. Post-layout simulated AC response of the proposed multiplier for $\mathrm{m}=0,1,2,3$.

13 In order to develop a deeper understanding of the design, the effect of process fabrication, power 14 supply and temperature variations on the circuit is studied through various simulations. The 
1 simulation results of $\mathrm{AC}$ response at different temperatures for $\mathrm{m}=0$ and $\mathrm{m}=3$ are shown in Fig. 9.

2 In these simulations $I_{X}$ is swept as an AC current source and $I_{Y}$ is set to $10 \mu \mathrm{A}$ and $40 \mu \mathrm{A}$ for $\mathrm{m}=0$ 3 and 3 respectively. The temperature is varied from $-30^{\circ} \mathrm{C}$ to $+70^{\circ} \mathrm{C}$ and the responses demonstrate 4 high robustness over $100^{\circ} \mathrm{C}$ temperature variations.
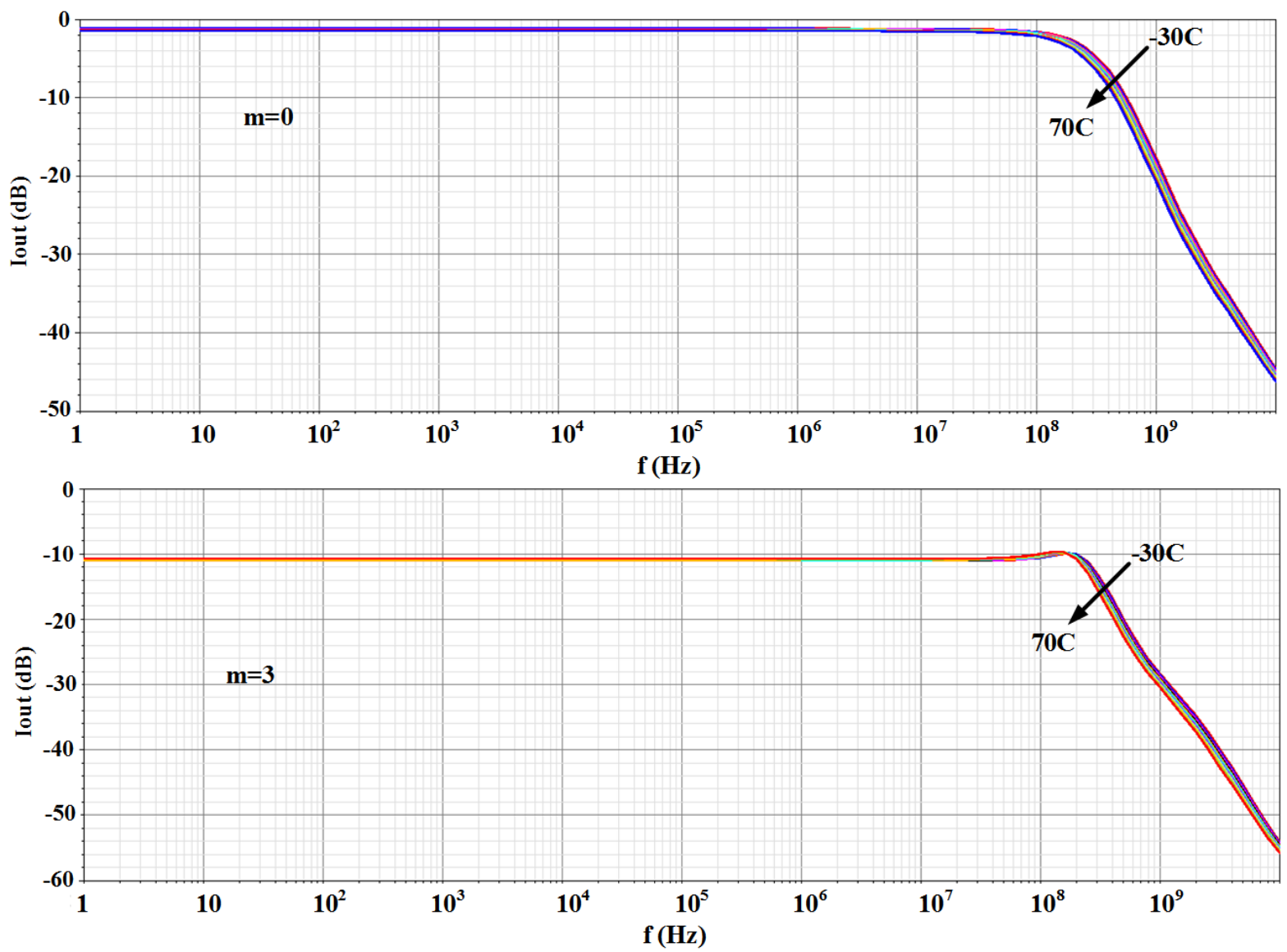

Fig. 9. Frequency response of the proposed multiplier versus temperature for $m=0$ and $m=3$

7 The effect of power supply variations on the output AC response is studied in Fig. 10, where the 8 power supply is swept between $1.6 \mathrm{~V}$ to $2 \mathrm{~V}( \pm 11 \%$ deviation from the nominal DC power supply 9 value, $1.8 \mathrm{~V}$ ) for $\mathrm{m}=0$ and $\mathrm{m}=3$. The input setup for this test is the same as for temperature analysis. 10 The results indicate that the circuit is highly insensitive to power supply fluctuations. This is 11 achieved thanks to the advantages of using translinear based current squarers. 

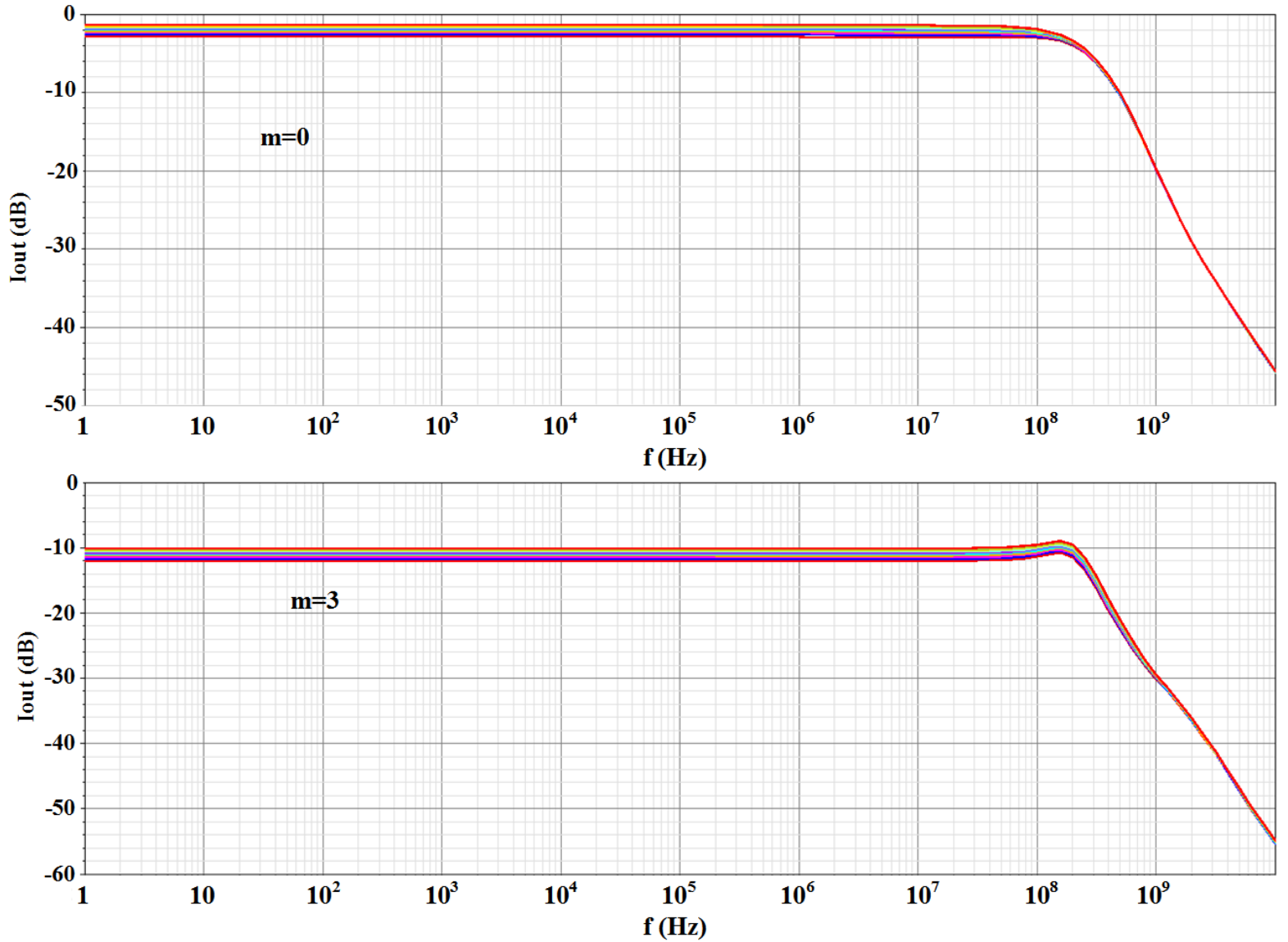

Fig. 10. Frequency response of the proposed multiplier versus power supply variations for $m=0$ and $m=3$

3 The effect of process corners in frequency response is shown in Fig.11. In this figure $I_{Y}$ is set to $410 \mu \mathrm{A}$ and $40 \mu \mathrm{A} \mathrm{DC}$ for $\mathrm{m}=0$ and $\mathrm{m}=3$ respectively while $I_{X}$ is set as a small signal current. The 5 AC response is simulated in fs, sf, ss and ff corners. The figure shows a high robustness against 6 process variations (less than a $2 \mathrm{~dB}$ difference over various corners in worst-case) which is achieved 7 thanks to the pure independent current mode output. 

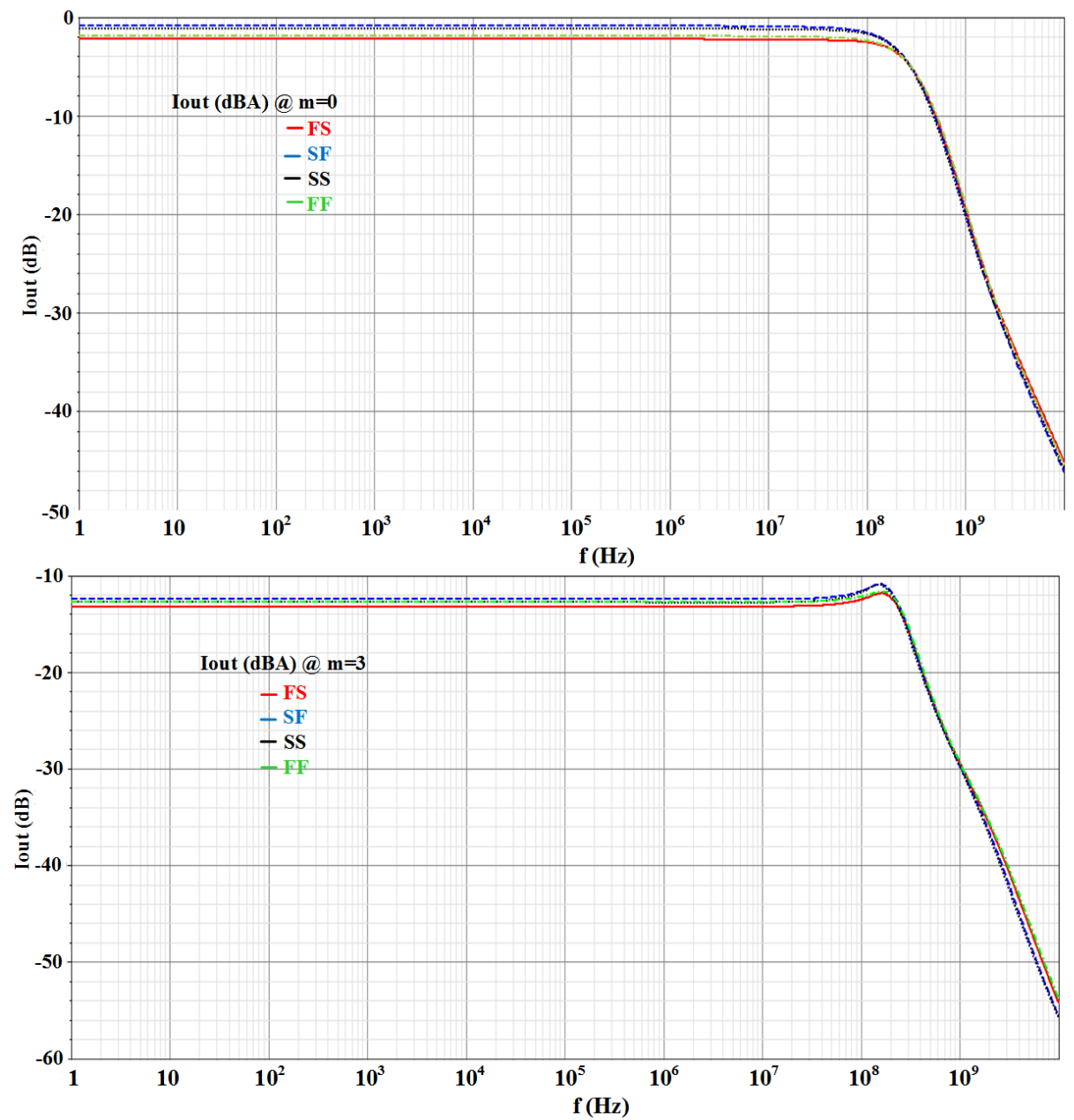

Fig. 11. Simulation of the frequency response for four different corners of $s f$, fs, ff and ss for $m=0$ and $m=3$

3 Monte Carlo analyses are carried out for bandwidth and total harmonic distortion to examine the 4 robustness of the circuit against process variations and mismatches. All Monte Carlo simulations 5 are performed with TSMC $0.18 \mu \mathrm{m}$ model and based on the MC section of technology file for both 6 process variations and mismatches.

7 Fig. 12 shows the Monte Carlo analyses of total harmonic distortion of the circuit for 1000 8 simulations for $\mathrm{m}=0$ and $\mathrm{m}=3$. In this simulations, the amplitude of each input current is set to 9 maximum $(10 \mu A$ for $\mathrm{m}=0$ and $40 \mu A$ for $\mathrm{m}=3)$ at $1 \mathrm{MHz}$ frequency. The results demonstrate a mean 10 value of 1.14 and 1.15 and a standard deviation of 0.05 and 0.07 for $\mathrm{m}=0$ and $\mathrm{m}=3$ respectively. 

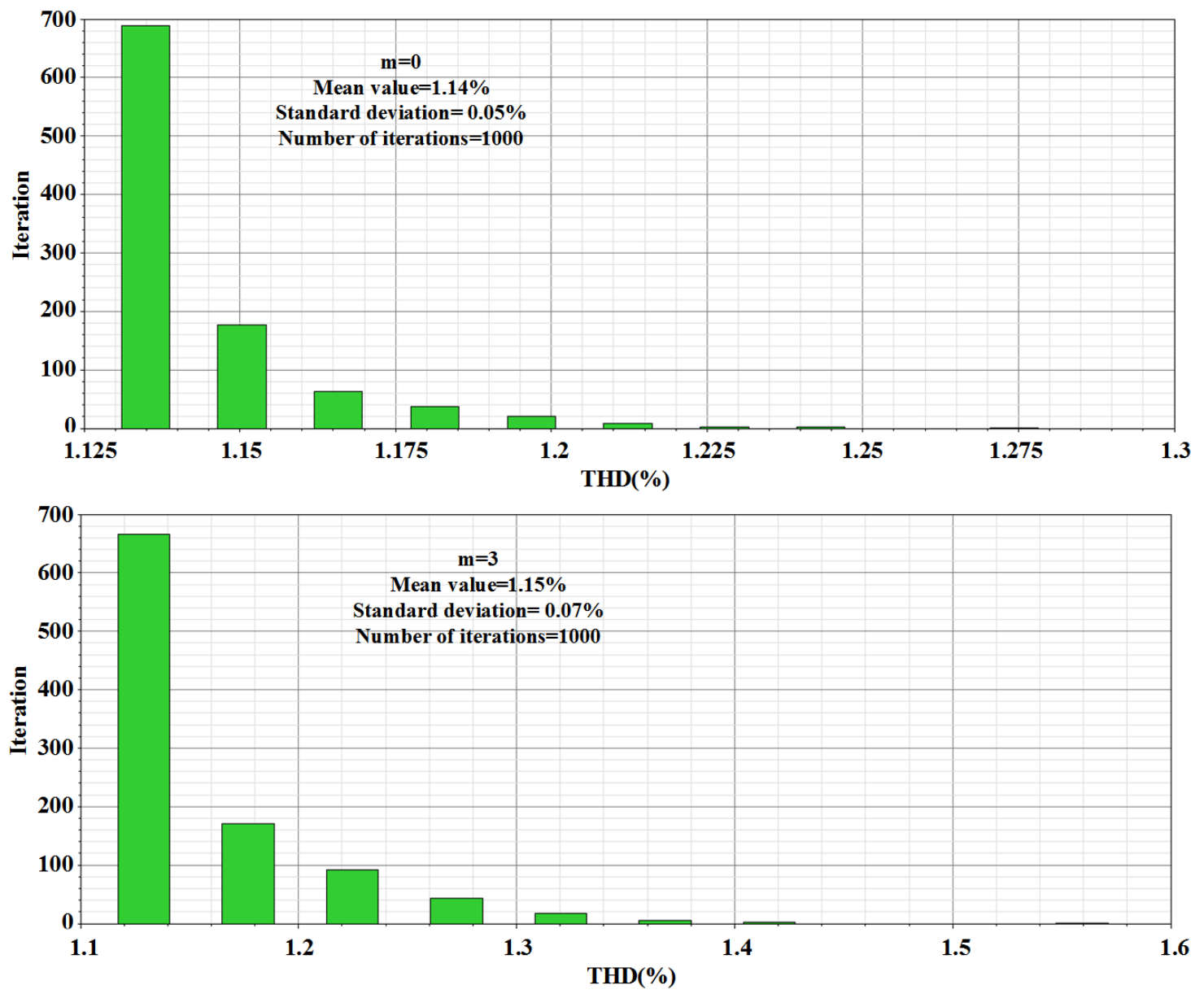

Fig. 12. Monte Carlo analyses of total harmonic distortion of the proposed multiplier for $m=0$ and $m=3$

3 The Monte Carlo analyses of $-3 \mathrm{~dB}$ bandwidth with 1000 iterations for $\mathrm{m}=0$ and $\mathrm{m}=3$ are shown in 4 Fig. 13. The values of the currents are the same as in Fig. 8. The mean value is $255.5 \mathrm{MHz}$ and $5 \quad 335.2 \mathrm{MHz}$ and the standard deviation is $9.45 \mathrm{MHz}$ and $7.42 \mathrm{MHz}$ for $\mathrm{m}=0$ and $\mathrm{m}=3$ respectively.

6 From Figures 12 and 13 it can be deduced that the proposed circuit is highly robust against possible 7 mismatches during manufacturing. This high level of robustness against mismatches is acquired 8 thanks to the TL based design. 

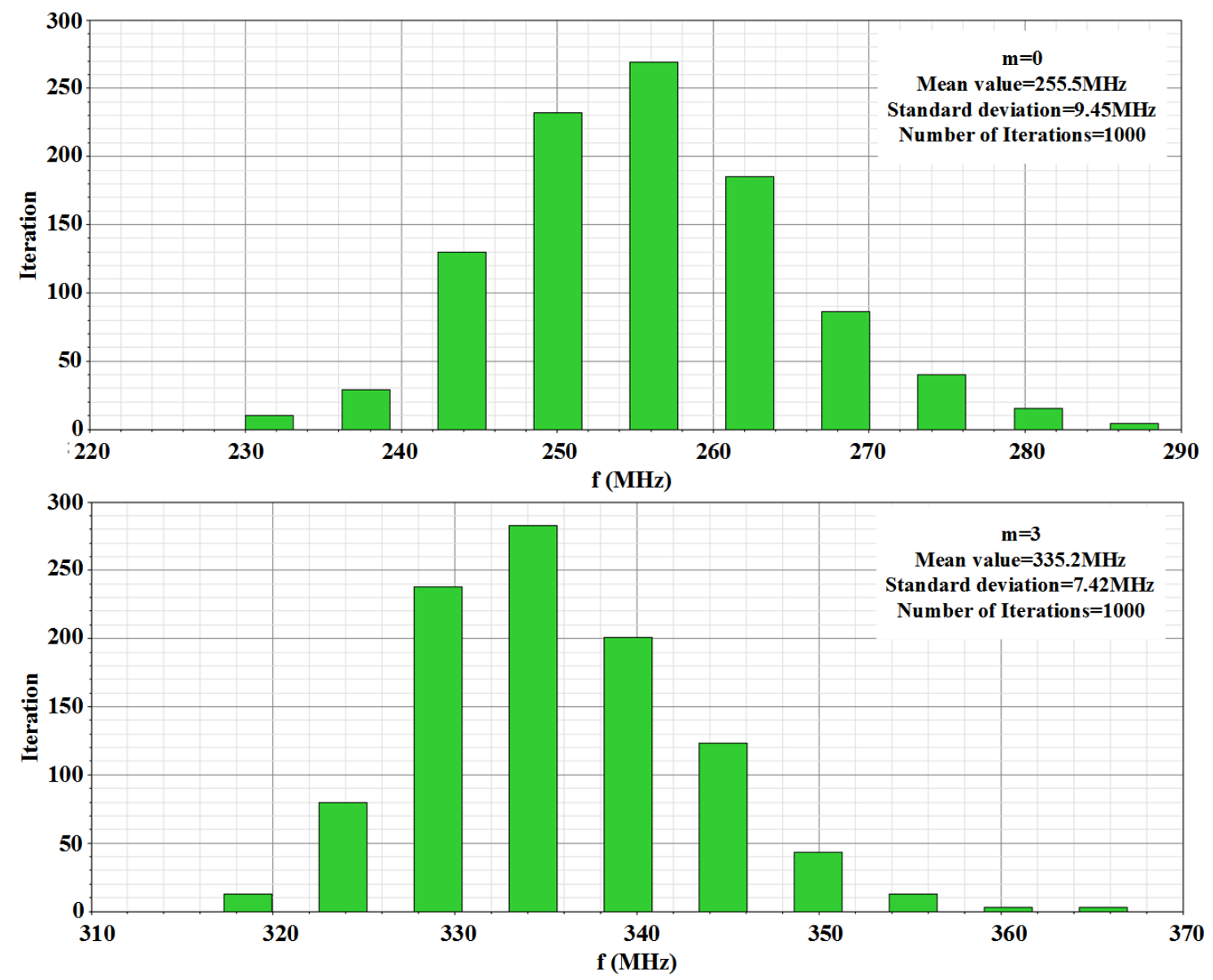

Fig. 13. Monte Carlo analyses of $-3 \mathrm{~dB}$ bandwidth of the proposed multiplier for $\mathrm{m}=0$ and $\mathrm{m}=3$

5. Conclusion

4 A new adjustable low power current-mode four-quadrant analog multiplier is proposed based on a 5 new current squarer circuit. The circuit allows the designers to adjust the input range of the 6 multiplier based on their own application without any increase in power consumption. The Post7 layout simulation plus Monte Carlo analyses endorsed the functionality and robustness of the 8 proposed multiplier against PVT variations. It is shown that THD and mismatch error of the circuit 9 are reduced as ' $\mathrm{m}$ ' is increased. The performance of the proposed multiplier is summarized and 10 compared to the other recently published works in Table 2 . The proposed multiplier is the only 11 reported one to offer an adjustable input range while improving the linearity and robustness against 12 PVT variations, yet keeping the bandwidth and power consumption at a reasonable level compared 13 to the other reported multipliers in a similar technology. 
Table 2. Summary of performance and comparison to prior works

\begin{tabular}{|c|c|c|c|c|c|c|c|c|c|c|c|}
\hline \multirow{2}{*}{ Ref./Year } & \multirow{2}{*}{$\begin{array}{c}{[22]} \\
/ 2003\end{array}$} & \multirow{2}{*}{$\begin{array}{c}{[8]} \\
/ 2009\end{array}$} & \multirow{2}{*}{$\begin{array}{c}{[23]} \\
/ 2010\end{array}$} & \multirow{2}{*}{$\begin{array}{r}{[24]} \\
/ 2011\end{array}$} & \multirow{2}{*}{$\begin{array}{c}{[9]} \\
/ 2012\end{array}$} & \multicolumn{2}{|c|}{$\begin{array}{l}{[25]} \\
/ 2014 \\
\end{array}$} & \multirow{2}{*}{$\begin{array}{c}{[15]} \\
/ 2015\end{array}$} & \multirow{2}{*}{$\begin{array}{c}{[3]} \\
/ 2016\end{array}$} & \multirow{2}{*}{$\begin{array}{c}{[11]} \\
/ 2017\end{array}$} & \multirow{2}{*}{ This work } \\
\hline & & & & & & Circuit1 & Circuit2 & & & & \\
\hline $\begin{array}{l}\text { Tech. } \\
(\mu \mathrm{m})\end{array}$ & 0.8 & 0.35 & 0.5 & 0.5 & 0.25 & 0.18 & 0.18 & 0.35 & 0.18 & 0.18 & 0.18 \\
\hline $\begin{array}{c}\text { Power } \\
(\mu \mathrm{W})\end{array}$ & 184 & 340 & 120 & 2000 & 168.1 & 60 & 75 & 232 & 89.2 & 150 & 186 \\
\hline $\begin{array}{c}\text { THD } \\
(\%)\end{array}$ & $\begin{array}{l}0.9 @ \\
1 \mathrm{KHz}\end{array}$ & $\begin{array}{l}0.97 @ \\
1 \mathrm{MHz}\end{array}$ & $\begin{array}{l}0.63 @ \\
10 \mathrm{KHz}\end{array}$ & $\begin{array}{c}0.2 @ \\
100 \mathrm{KHz}\end{array}$ & $\begin{array}{l}0.91 @ \\
1 \mathrm{MHz}\end{array}$ & NA & NA & $\begin{array}{c}0.16 \\
@ 1 \mathrm{MHz}\end{array}$ & $\begin{array}{l}1.01 @ \\
1 \mathrm{MHz}\end{array}$ & $\begin{array}{l}0.8 @ \\
1 \mathrm{MHz}\end{array}$ & $\begin{array}{c}1 @ \\
1 \mathrm{MHz}\end{array}$ \\
\hline $\begin{array}{c}\text { Supply } \\
\text { Voltage (V) }\end{array}$ & 1.5 & 3.3 & 3 & 3.3 & 2.5 & 1.2 & 1.2 & 2 & 1.8 & \pm 0.75 & 1.8 \\
\hline $\begin{array}{c}\text { Bias } \\
\text { current }(\mu \mathrm{A})\end{array}$ & 6 & 10 & 10 & 40 & 10 & 40 & 40 & 4.6 & 10 & 10 & 10 \\
\hline $\begin{array}{c}\text { Input } \\
\text { range }(\mu \mathrm{A})\end{array}$ & 12 & \pm 10 & \pm 10 & \pm 20 & \pm 10 & $0-10$ & $0-10$ & \pm 10 & \pm 20 & \pm 10 & $\underset{\mathrm{m}: 0-3}{ \pm(1+\mathrm{m}) \times 10}$ \\
\hline $\mathrm{BW}(\mathrm{MHz})$ & 5.5 & 41.8 & 18 & 3 & 278 & 79.6 & 59.7 & 485 & 840 & 300 & 332.3 \\
\hline $\begin{array}{c}\mathrm{N}^{\circ} \text { of } \\
\text { quadrants }\end{array}$ & 4 & 4 & 2 & 2 & 4 & 1 & 1 & 4 & 4 & 4 & 4 \\
\hline Sim/Meas & Meas. & Sim. & Meas. & Meas. & Sim. & Sim. & Sim. & Sim. & Sim. & Sim. & $\begin{array}{c}\text { Post } \\
\text { Layout }+\mathrm{MC}\end{array}$ \\
\hline
\end{tabular}

3 The authors thank Dr. Ali Sahafi for his valuable comments and suggestions during this work.

\section{References}

5 [1] G. Han and E. Sanchez-Sinencio, "CMOS transconductance multipliers: A tutorial," IEEE Transactions on Circuits and Systems II: Analog and Digital Signal Processing, vol. 45, no. 12, pp. 1550-1563, 1998.

[2] D. Coue and G. Wilson, "A four-quadrant subthreshold mode multiplier for analog neural-network applications," IEEE transactions on neural networks, vol. 7, no. 5, pp. 1212-1219, 1996.

[3] A. Baharmast, S. J. Azhari, and S. Mowlavi, "A new current mode high speed four quadrant CMOS analog multiplier," in Electrical Engineering (ICEE), 2016 24th Iranian Conference on, 2016, pp. 1371-1376: IEEE.

[4] C.-C. Chang and S.-I. Liu, "Weak inversion four-quadrant multiplier and two-quadrant divider," Electronics Letters, vol. 34, no. 22, pp. 2079-2080, 1998.

[5] C. Sawigun and W. Serdijn, "Ultra-low-power, class-AB, CMOS four-quadrant current multiplier," Electronics Letters, vol. 45, no. 10, pp. 483-484, 2009.

[6] A. Mahmoudi, A. Khoei, and K. Hadidi, "A novel current-mode micropower four quadrant CMOS analog multiplier/divider," in Electron Devices and Solid-State Circuits, 2007. EDSSC 2007. IEEE Conference on, 2007, pp. 321-324: IEEE.

[7] A. J. Lopez-Martin and A. Carlosena, "Design of MOS-translinear multiplier/dividers in analog VLSI," VLSI Design, vol. 11, no. 4, pp. 321-329, 2000. 
[8] A. Naderi, A. Khoei, K. Hadidi, and H. Ghasemzadeh, "A new high speed and low power fourquadrant CMOS analog multiplier in current mode," AEU-International Journal of Electronics and Communications, vol. 63, no. 9, pp. 769-775, 2009.

[9] A. Alikhani and A. Ahmadi, "A novel current-mode four-quadrant CMOS analog multiplier/divider," AEU-International Journal of Electronics and Communications, vol. 66, no. 7, pp. 581-586, 2012.

[10] K. Tanno, O. Ishizuka, and Z. Tang, "Four-quadrant CMOS current-mode multiplier independent of device parameters," IEEE Transactions on Circuits and Systems II: Analog and Digital Signal Processing, vol. 47, no. 5, pp. 473-477, 2000.

[11] I. Aloui, N. Hassen, and K. Besbes, "A CMOS current mode four quadrant analog multiplier free from mobility reduction," AEU-International Journal of Electronics and Communications, 2017.

[12] A. Ravindran, K. Ramarao, E. Vidal, and M. Ismail, "Compact low voltage four quadrant CMOS current multiplier," Electronics letters, vol. 37, no. 24, pp. 1428-1429, 2001.

[13] I. Navarro, A. J. López-Martín, C. A. De La Cruz-Blas, and A. Carlosena, "Very Low Voltage CMOS Current Multiplier with Floating DC Level Shifters," Analog Integrated Circuits and Signal Processing, vol. 37, no. 3, pp. 265-268, 2003.

[14] P. Prommee, M. Somdunyakanok, M. Kumngern, and K. Dejhan, "Single low-supply current-mode CMOS analog multiplier circuit," in Communications and Information Technologies, 2006. ISCIT'06. International Symposium on, 2006, pp. 1101-1104: IEEE.

[15] N. Beyraghi and A. Khoei, "CMOS design of a low power and high precision four-quadrant analog multiplier," AEU-International Journal of Electronics and Communications, vol. 69, no. 1, pp. 400407, 2015.

[16] E. Seevinck and R. J. Wiegerink, "Generalized translinear circuit principle," IEEE Journal of SolidState Circuits, vol. 26, no. 8, pp. 1098-1102, 1991.

[17] C. De La Cruz-Blas, G. Thomas-Erviti, J. Algueta-Miguel, and A. López-Martín, "CMOS analogue current-mode multiplier/divider circuit operating in triode-saturation with bulk-driven techniques," Integration, the VLSI Journal, vol. 59, pp. 243-246, 2017.

[18] R. Vargas-Bernal and A. Carlos, "A survey on the static and dynamic translinear paradigm," in Integrated Circuits for Analog Signal Processing: Springer, 2013, pp. 289-306.

[19] L. Safari and S. J. Azhari, "A novel low voltage very low power CMOS class AB current output stage with ultra high output current drive capability," Microelectronics Journal, vol. 43, no. 1, pp. 34-42, 2012.

[20] B. Razavi, Design of analog CMOS integrated circuits, Second Edition ed. McGraw-Hill Education, 2015.

[21] G. Palmisano, G. Palumbo, and S. Pennisi, "Harmonic distortion on class AB CMOS current output stages," IEEE Transactions on Circuits and Systems II: Analog and Digital Signal Processing, vol. 45, no. 2, pp. 243-250, 1998.

[22] C. De La Cruz-Blas, A. Lopez-Martin, and A. Carlosena, "1.5 V four-quadrant CMOS current multiplier/divider," Electronics letters, vol. 39, no. 5, pp. 434-436, 2003.

[23] A. J. Lopez-Martin, A. Carlos, J. Ramirez-Angulo, and R. G. Carvajal, "Compact low-voltage CMOS current-mode multiplier/divider," in Circuits and Systems (ISCAS), Proceedings of 2010 IEEE International Symposium on, 2010, pp. 1583-1586: IEEE.

[24] J. M. A. Miguel, A. Carlos, and A. J. Lopez-Martin, "Fully differential current-mode CMOS triode translinear multiplier," IEEE Transactions on Circuits and Systems II: Express Briefs, vol. 58, no. 1, pp. 21-25, 2011.

[25] C. Popa, "Improved accuracy current-mode multiplier circuits with applications in analog signal processing," IEEE transactions on very large scale integration (VLSI) systems, vol. 22, no. 2, pp. 443-447, 2014. 